\title{
Leprosy patients in Bengaluru, India: associated comorbidities, costs for management and linkages to welfare schemes
}

\author{
PRIYANKA RANGANATHA ${ }^{\mathrm{a}}$, \\ SHARATH BURUGINANAGARAJA ${ }^{\text {a }}$ \& \\ RAGUNATHA SHIVANNA ${ }^{\mathrm{a}}$ \\ ${ }^{\mathrm{a}}$ ESIC Medical College and PGIMSR, Rajajinagar, Bangalore, India
}

Accepted for publication 21 November 2019

\begin{abstract}
Summary
Introduction: India accounts for the $60 \%$ of the global burden of leprosy. We conducted a study to identify the various comorbid conditions, costs incurred for its management and the status of linkages to public welfare schemes amongst leprosy patients admitted at a leprosy hospital in Bengaluru, India.

Methods: A cross sectional study was conducted between March and July, 2018, at a leprosy hospital in Bengaluru, India. All the admitted patients were interviewed using a semi-structured questionnaire. The quantitative data was double entered using EpiData data entry software and was analysed using EpiData analysis software for numbers, proportions and range.

Results: A total of 80 admitted in-patients were interviewed of which the male to female ratio was $3: 1$. A majority $(90 \%)$ of them had comorbid conditions, including neuropathic ulcers, diabetes mellitus, hypertension, mental illnesses and cellulitis. Most of them had incurred some expenses for morbidity management. Only half of the patients were found to be linked to public social welfare schemes.

Conclusion: Leprosy is associated with comorbid conditions and as a policy all diagnosed and registered patients under the programme should be screened for diabetes mellitus, hypertension, HIV and mental illnesses at regular intervals. Efforts are to be made to integrate the primary health care services and bring down the patients costs on comorbidities.
\end{abstract}

Keywords: Leprosy, comorbidities, India, linkages

\section{Introduction}

Despite global efforts, leprosy is still a public health problem. More than 200,000 new cases of leprosy are registered worldwide annually, with $60 \%$ occurring in India alone. In India, $551(83 \%)$ districts have attained the levels of elimination (less than one case per ten thousand population). ${ }^{1}$ The country has 88,000 cases with the prevalence rate of $0.69 / 10,000(2015-16)$

Correspondence to: Dr Sharath BN, Department of Community Medicine, ESIC Medical College and PGIMSR, Rajajinagar, Bangalore-560010, India (e-mail: sharathbn@yahoo.com) 
and the trends shows a decline. ${ }^{1,2}$ The state of Karnataka in southern India has 2,726 leprosy cases of which 2,136 (78\%) are multi-bacillary (MB) and $989(12 \%)$ are pauci-bacillary (PB) cases. $^{1}$

The unprecedented challenge that persists for leprosy patients is the development of comorbidities during treatment due to reactivation or interaction with other asymptomatic infections. ${ }^{3}$ There has been evidence for the presence of co-morbidities such as chronic hepatitis, latent tuberculosis, chronic strongyloidiasis, Chagas' disease, diabetes mellitus and human immunodeficiency virus (HIV). ${ }^{4}$ The other co-morbidities include neuropathic ulcers, hearing impairment, mental disorders, restless leg syndrome and renal amyloidosis. ${ }^{4,5}$

Leprosy patients are often stigmatised by society. A majority of severe or full blown cases do not have a regular source of income and are dependent on others. Many of them are forced to leave their jobs due to the long duration of treatment, disabilities, the development of comorbidities and the prevailing social stigma and discrimination. ${ }^{6}$ Often, they are displaced from their families and are bound to depend on the government or non-governmental organizations (NGOs) for the costs incurred in the management of comorbidities. The Government of India (GoI) offers many social welfare schemes which aim to benefit the people below the poverty line (BPL) and there are several NGO programmes for the deprived which also facilitate linkages to promote good quality of life amongst this group. ${ }^{7,8}$ It is important to understand whether marginalised populations, such as leprosy patients, are availing such schemes. It is speculated that many patients might be ignorant about the government schemes and would not have benefitted. Currently, the information on the associated co-morbidities of leprosy patients, the costs incurred for its management and the status of linkages to Government social welfare schemes remain sparse. Hence, we conducted a study among the leprosy patients admitted in a Government leprosy hospital, Bengaluru in India to (a) determine the proportion of co-morbidities present among the patients (b) costs incurred for the management of their comorbid conditions, and (c) to ascertain the status of linkages to any of the public social welfare schemes.

\section{Methods}

A cross sectional study was conducted during the months of March to July, 2018 at a leprosy hospital, Bengaluru which is a public tertiary health care facility and specifically caters to leprosy patients through out-patient and in-patient services. The hospital has sixty beds with separate wards for both genders and has full occupancy rate throughout the year. The specialized services offered include the consultation services from physician, surgeon, dermatologist and physiotherapist. The facility is equipped with operation theatre and a laboratory to assess the slit skin smears.

For the purpose of the study, we included all those leprosy patients who were admitted at the inpatient facilities during the study period. Informed consent was obtained from the patients (or their guardians in case the patient could not provide consent due to mental illness), after explaining in detail to them the purpose, procedure and the utility of the study in their local vernacular language. The identities of the study participants were kept confidential.

The data were collected by the principal investigator using a semi-structured questionnaire after pilot testing the questionnaire. The general information about the patients and their associated comorbidities were obtained from the case records and reports while the information regarding the costs and linkages were obtained by personal interview at a time and place convenient to the patient. 
The variables collected included age, sex, address, education, type of family, dependency, per capita income, type of leprosy, duration of the diseases, comorbidities, costs incurred for pre-existing and existing comorbidities, treatment history for the comorbid condition and status of linkages to social welfare schemes.

The operational definition for costs incurred included any amount spent on diagnosis or treatment or medications for a comorbid condition during the last six months from the date of interview. It did not include the indirect costs borne by the patients or their family members.

The Institutional ethics approval was obtained from the ESIC Medical College and PGIMSR, Bengaluru. The administrative permission for the conduct of the study was obtained from the Director, Leprosy hospital and the State Leprosy Officer, Karnataka. The data from each patient were double-entered, validated, appended and analysed using EpiData v. 3.1 for entry and validation and v. 2.2.2.183 for appending and analyses (EpiData Association, Odense, Denmark). The data were analysed for means, proportions and range.

\section{Results}

A total of 80 admitted leprosy patients were interviewed during the study period.

\section{SOCIO-DEMOGRAPHIC CHARACTERISTICS (TABLE 1)}

Of the 80 patients, 60 (75\%) were males and 20 (25\%) were females; 45 (55\%) had multibacillary leprosy and 35 (45\%) had paucibacillary leprosy. Among them, 40 (50\%) were aged above 65 years and 19 (24\%) belonged to the age group of 55-64 years. A majority (64\%) were from rural areas and half of them were illiterates. $70 \%$ of patients were unskilled workers prior to the disease and currently $89 \%$ of them were unemployed. During the course of their disease, $40 \%$ of them became separated or divorced. Most patients were solely dependent on the public welfare schemes $(40 \%)$ or their children $(36 \%)$ for their livelihood. According to the modified Kuppuswamy classification (2018), a majority (80\%) belonged to the lower class and $42 \%$ were not covered under any Government social welfare or protection schemes. The median time since the study participants were diagnosed with leprosy was found to be 20 years (range 3 months to 60 years). The twelve months or more of treatment had been completed by $56 \%$ of patients.

\section{COMORBIDITIES}

A majority (91\%) of the patients had comorbidities and they were found to be neuropathic ulcers $74 \%$ (59), hypertension 39\% (31), diabetes mellitus 15\% (12), ocular disorders 15\% (12), psychiatric illness $13 \%$ (10) and cellulitis $11 \%(9)$. While most of the patients had multiple comorbidities, 34\% (27) had neuropathic ulcers alone. The status of HIV screening was not mentioned in the case records accessed.

\section{COST FOR DISEASE MANAGEMENT}

The information regarding the cost incurred for the management of the disease and their comorbidities were collected for the past six months. The main drivers for out of pocket expenditure were related to transportation, investigations and medications. The mean cost incurred for transportation were INR 224 (Range: 50-4,000) and for investigations and medications were INR 1000 (Range: 0-30000). The patients had availed both public and private health facilities in the process of their disease management. 
Table 1. Sociodemographic characteristics of leprosy patients admitted at the Leprosy Hospital, Bengaluru, India. $(N=80)$

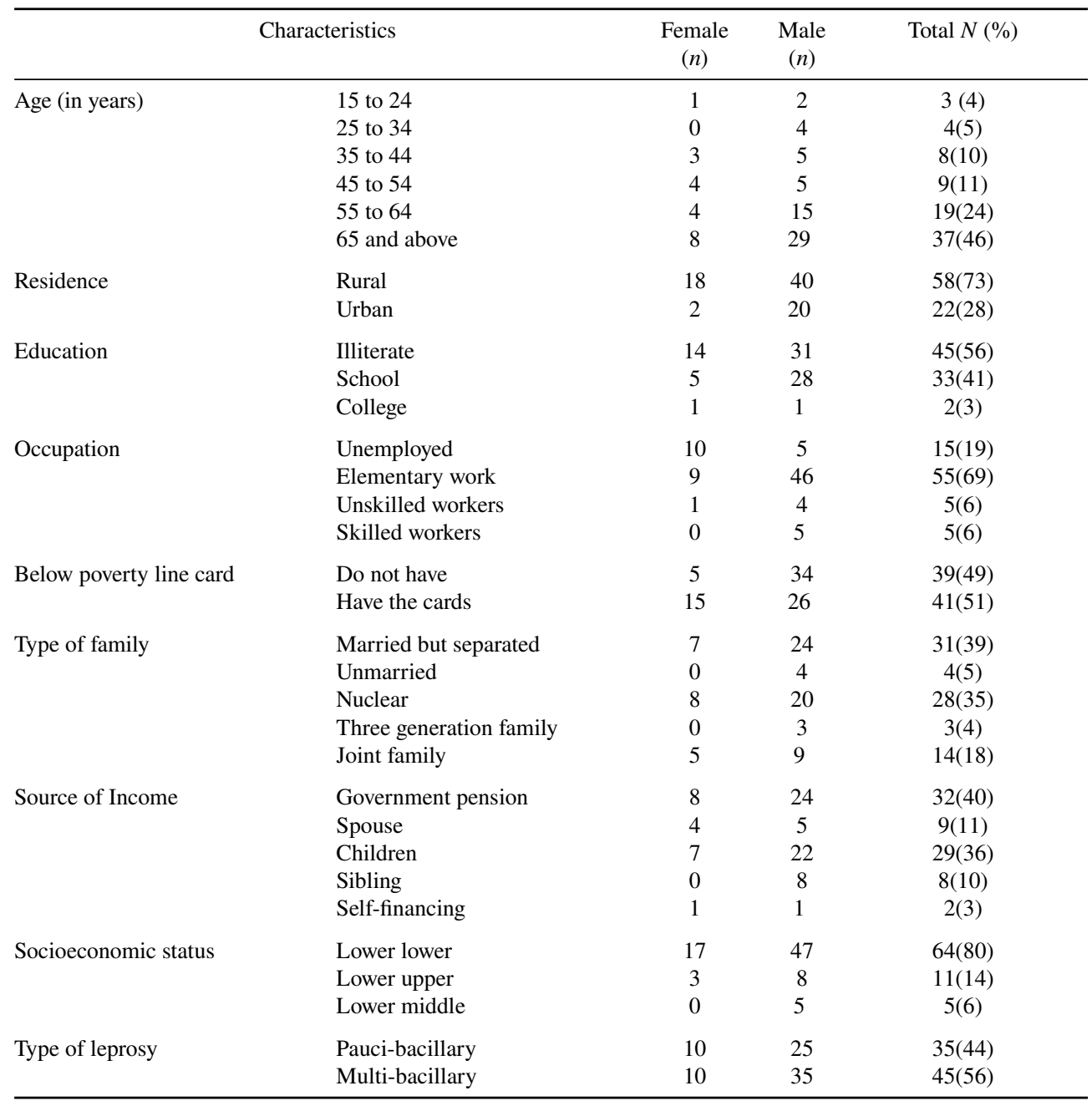

\section{LINKAGES TO SOCIAL WELFARE SCHEMES}

Nearly $51 \%$ (41) patients had the below poverty line card to collect raw food grains issued through the public distribution system from the Government welfare schemes. These patients were admitted at the hospital for a longer than average duration and the health facility provided the food during their stay.

The leprosy patients were asked about availing any social welfare schemes facilitated through NGOs during their disease period and it was found that only one patient was scheduled for reconstructive surgery through an NGO. 


\section{Discussion}

This is one of the first studies conducted in India to determine the associated comorbidities, costs incurred for disease management and linkages to Government welfare schemes amongst leprosy patients. Our study reveals that $91 \%$ of leprosy patients suffer from comorbidities and are aged above 55 years; they incur out of pocket expenditure for their comorbidity management and only half of the patients avail of basic social welfare schemes. The leprosy patients were not subjected to HIV screening.

Our study suggests that three in four patients were male and many were aged above 65 years and were from rural areas. The influx of patients from rural areas may be possibly due to lack of facilities to admit the patients for longer duration or non-availability of services for comorbidities and its complications. Also, a majority of the patients were from lower socioeconomic section due to which they would have not afforded the services from private health facilities. It is also seen that most of the patients have incurred costs for disease management of which most were spent on investigations and medications. We observed that many patients were separated from their family or were divorcees and this spurge in separation compared to general population could be a proxy indicator to the existence of stigma prevailing in society.

Of all the comorbidities, neuropathic ulcers were the commonest form observed and prompted admission to the hospital for the surgical care of the wound or ulcer. This indicates that the patients were not adequately guided or educated regarding the care of wounds or their complications at the time of diagnosis or during the course of treatment. Appropriate and timely health education with regular follow-up could have halted the progression of the disease. About $15 \%$ patients were also suffering from hypertension or diabetes mellitus and the existence of these diseases further complicates healing. Nearly one in ten patients suffer from either ocular comorbidities, mental illnesses or cellulitis and this group of patients require specialised care and regular follow-up.

In India, the public health services are provided free of cost to the patients. The costs incurred for the management of the comorbid conditions indicate that there is a tendency of patients to seek health services from the private health sector as they might have felt unsatisfied with the services provided at primary or secondary public health facilities. ${ }^{9}$

The central and state governments offer various social welfare schemes for downtrodden people. ${ }^{10,11}$ In general, the schemes include providing food grains to supplement nutrition and providing a disability pension. ${ }^{12}$ More than half of the patients have availed nutrition schemes where the patients are provided with food grains and other cooking materials every month through the public distribution system; however, many of the patients are neither aware nor eligible for such schemes. Leprosy patients with disability Grade 1 and 2 receive allowances every month. ${ }^{13}$ Apart from that, eligible leprosy patients would be provided with footwear and offered services for reconstructive surgeries. ${ }^{13}$ The National Leprosy Elimination Programme (NLEP) has various schemes for NGOs to facilitate the linkage of leprosy patients from the community to the health care facilities; however, there is little uptake of such schemes among NGOs in the districts, for reasons not known. ${ }^{14}$

Our study findings have the following programmatic implications. First, all the leprosy patients who are diagnosed should be registered as a cohort and screened for HIV, diabetes mellitus and hypertension regularly at every three months, irrespective of the patients being in treatment or released from treatment. Second, the diagnosed leprosy patients should be educated and empowered to undertake self-care for the expected complications. ${ }^{15}$ The usage of innovative information technology enabled tools should be leveraged to enhance patient skills for management. Third, continuous efforts should be made by the programme to drive 
away the stigma associated with leprosy in the community. ${ }^{16}$ Leprosy awareness generation among school children by incorporating a chapter on leprosy in their text books will benefit the disease elimination in the long run. ${ }^{17}$ There is also a need to conduct leprosy awareness among the population in a campaign mode through active case finding. Fourth, the involvement of NGOs is crucial. The programme should develop viable mechanisms to engage NGOs and try to fill the gaps in the existing diagnosis, treatment and rehabilitation pathways. The focus on patients' vocational rehabilitation will help the patients to live an independent life.

The study has strengths and limitations. The strengths are it reflects the ground reality as the information is based on the patients who had suffered from leprosy and its comorbidities for quite a long duration. The limitation is that we could not ascertain the exact duration of onset of comorbidities in these patients. The calculation of costs includes only the direct costs incurred by the patients and we could not estimate the indirect costs. The findings are specific to this region and generalisation of the findings must be done with caution.

To conclude, leprosy is associated with comorbidities, either related to the disease itself or to other common infectious or non-communicable conditions. The patients incur expenditure for the management of comorbid conditions, which could be minimised by integrating and strengthening the primary health care services. The involvement of NGOs is needed to facilitate and reduce the accessibility gap between the patients and the health systems.

\section{Acknowledgements}

We acknowledge the support and permission rendered by Dr Rajni, State Leprosy Officer, Government of Karnataka for the conduct of the study. We also thank the Director and staff of the Leprosy Hospital, Magadi Road, Bengaluru for their cooperation. We wholeheartedly express our gratitude to all the leprosy patients who took part in the study.

\section{Authors' contributions:}

SBN, PK and RS conceived the idea. SBN and RS obtained the permission to conduct the study and obtained ethics approval. PK, SBN and RS collected the data. SBN and PK analysed the data. SBN wrote the first draft. RS and PK provided their inputs and final approval for submission.

\section{Conflict of interest:}

None

\section{References}

1 Central Leprosy Division. NLEP Annual Report 2015-2016. National Leprosy Eradication Programme. 2016; Annual Rep. [Internet]. Available from: http://www.fareshare.org.uk/wpcontent/uploads/2016/09/FareShare_Annual_Report_2016_Interactive.pdf.

2 Rao PN, Suneetha S. Current situation of leprosy in India and its future implications. Indian Dermatology Online Journal, 2018 [cited 2019 Jun 21]; 9(2):83. [Internet]. Available from: http://www.ncbi.nlm.nih.gov/pubmed/29644191.

3 Bilodeau M, Burns S, Gawoski J, Moschella S, Ooi W. Co-morbid infections in Hansen's disease patients in the United States: considerations for treatment. Am J Trop Med Hyg, 2013; 89(4): 781-783, [Internet]. Available from: http://www.ncbi.nlm.nih.gov/pubmed/23980128.

4 Saraya MA, Al-Fadhli MA, Qasem JA. Diabetic status of patients with leprosy in Kuwait. Journal of Infection and Public Health, 2012; 5(5): 360-365, [Internet]. Available from: http://dx.doi.org/10.1016/j.jiph.2012.08.001.

5 Ozturk S, Ozturk T, Can I. Renal involvement in leprosy: evaluation of patients in Turkey. 2017; 10145. 
6 Stevelink SAM, Van Brakel WH, Augustine V. Stigma and social participation in Southern India: Differences and commonalities among persons affected by leprosy and persons living with HIV/AIDS. Psychology, Health and Medicine, 2011 [cited 2019 Jun 21]; 16 (6): 695-707. [Internet]. Available from: https://pdfs. semanticscholar.org/46eb/c2ed9f7975bb27d46b628c8a4b71805c1f6c.pdf?_ga=2.167895649.1563064280. 1561138589-205220349.1561138589.

7 NationalLeprosyEradicationProgramme. Guidelines for Facilitating Reconstructive Surgery in Leprosy. 2007; 2-8. Available from: http://www.iapsmgc.org/userfiles/1GUIDELINES_ON_DISABILITY_PREVENTION_ AND_REHABILITATION_LEPROSY.pdf.

8 Leprosy N. Programme E. National Leprosy Eradication Programme NGO Scheme Year 2013. 2013.

9 Tiwari A, Suryawanshi P, Raikwar A, Arif M, Richardus JH. Household expenditure on leprosy outpatient services in the Indian health system: A comparative study. PLoS Neglected Tropical Diseases, 2018 [cited 2019 Jun 21]; 12 (1): e0006181. [Internet]. Available from: http://www.ncbi.nlm.nih.gov/pubmed/29300747.

10 Social Welfare Department. Leprosy welfare scheme/Official Website of Chhattisgarh Social Welfare Department, Government of Chhattisgarh, India. [cited 2019 Jun 21]. [Internet]. Available from: https://sw.cg.gov.in/en/leprosy-welfare-scheme.

11 Kapil U, Chaturvedi S, Nayar D. National nutrition supplementation programmes. Indian Pediatrics, 1992 Dec [cited 2019 Jun 21]; 29 (12): 1601-1613. [Internet]. Available from: http://www.ncbi.nlm.nih.gov/pubmed/1291517.

12 NITI Aayog. Nourishing India. New Delhi; 2019 [cited 2019 Jun 21]. [Internet]. Available from: https://niti.gov.in/writereaddata/files/document_publication/Nutrition_Strategy_Booklet.pdf.

13 Programme Implementation Plan (PIP) for 12 Plan Period CENTRAL LEPROSY DIVISON Directorate General of Health Services Ministry of Health \& Family Welfare. 2012 [cited 2019 Jun 21]; 1-67. Available from: http://nlep.nic.in/pdf/FinalPIP, on 3 May 2013.pdf.

14 Leprosy N, Programme E. National Leprosy Eradication Programme NGO Scheme Year 2013. 2013 [cited 2019 Jun 21]; Available from: http://nlep.nic.in/pdf/NGOscheme-2013.pdf.

15 Butlin CR. The challenge of Multimorbidity in the context of leprosy. Lepr Rev, 2016; 87(2): 151-157, [Internet]. Available from: https:/www.lepra.org.uk/Platforms/Lepra/Files/lr/June16/16-0006.pdf.

16 Central Leprosy Division. NLEP-guidelines on reduction of stigma and discrimination against persons affected by leprosy. Health (San Francisco). [cited 2019 Jun 21]; 1-10. [Internet]. Available from: http://nlep.nic.in/pdf/Guidelines - reduction of stigma.pdf.

17 Cariappa M. Leprosy detection: Involvement of teachers. Indian Journal of Dermatology, Venereology and Leprology, 2009 [cited 2019 Jun 21]; 73 (4): 266. [Internet]. Available from: http://www.ncbi.nlm.nih.gov/pubmed/17675740. 